\title{
Modificação da Fibra de Coco com Polianilina e o seu Uso como Sensor de Pressão
}

\author{
Fernando G. de Souza Jr, Luciana 0. Paiva \\ Laboratório de Biopolímeros e Sensores, IMA/UFRJ \\ Ricardo C. Michel \\ Laboratório de Físico-Química de Polímeros e Instrumentação, IMA/UFRJ \\ Geiza E. de Oliveira \\ Departamento de Química, UFES
}

Resumo: O presente trabalho teve por foco a modificação de fibras de coco, tornando-as materiais compósitos condutores de eletricidade. Para tanto, foi utilizada uma técnica de polimerização in situ, capaz de gerar nanopartículas de polianilina sobre a superfície de fibras tratadas. As fibras modificadas com polianilina foram caracterizadas por FTIR, UV-Vis, DRX e SAXS. Além disso, foram submetidas a ensaios eletromecânicos, com a finalidade de indicar quais condições de modificação são mais eficientes para a obtenção de um material sensível a esforços compressivos. Os resultados de sensibilidade a compressão sugerem que os materiais modificados com as menores quantidades de polianilina são os mais adequados para aplicações no campo de sensores de pressão de natureza resistiva, podendo ser utilizados no desenvolvimento de dispositivos inteligentes.

Palavras-chave: Condutividade elétrica, fibras de coco, polianilina, sensibilidade a compressão.

\section{Coconut Fibers Modified with Polyaniline Nanoparticles Are Used in Pressure Sensor}

Abstract: Coconut fibers were covered with polyaniline in order to obtain electrically conductive composite materials. For this purpose aniline was polymerized in the presence of coconut fibers, leading to the formation of polyaniline nanoparticles over the surface of the fibers. The treated fibers were analyzed by FTIR, UV-Vis, DRX and SAXS, being also submitted to electromechanical assays, in order to find the best conditions for obtaining materials with optimal compressional sensitivity. The results suggest that materials obtained with smaller amounts of polyaniline are the most adequate for applications as resistive pressure sensors. This class of materials can be used in the development of intelligent devices. Keywords: Electrical conductivity, coconut fibers, polyaniline, sensitivity to compress.

\section{Introdução}

O coco verde vem sendo intensamente explorado por ser um isotônico natural, sendo ideal para repor o líquido perdido após atividades físicas, ou até mesmo em casos de desidratação mais severa. Dadas às proporções, o consumo deste produto natural gera um grande impacto ambiental, relacionado com o descarte final das suas cascas ( 4 MTon/ano), as quais representam de 80 a $85 \%$ de seu peso bruto $^{[1]}$. Além do elevado volume, estas cascas, quando descartadas, demandam mais de oito anos para a completa decomposição, uma vez que são pobres em hemicelulose e ricas em lignina e celulose ${ }^{[2,3]}$.

Dadas as suas excelentes características, as fibras de coco são muito utilizadas na indústria automobilística, principalmente na forração de bancos, onde apresentam significativas vantagens em relação à espuma plástica, uma vez que são inodoras, resistentes à umidade, não são atacadas por roedores, não sofrem decomposição microbiana, e possuem uma baixa condutividade térmica $(\sim 0,044 \mathrm{~W} / \mathrm{mK})^{[4]}$.

As vantagens apresentadas até este ponto já tornam o estudo deste tipo de fibra muito interessante. Entretanto, este estudo pode se tornar ainda mais atrativo, através de modificações que permitam usos mais nobres para este material, agregando valor ao mesmo. Uma alternativa de modificação bastante elegante consiste na modificação da superfície da fibra via polimerização. O polímero sintetizado sobre a superfície da fibra está intimamente ligado à mesma e, se adequadamente escolhido, pode ser usado como um recobrimento inteligente, capaz de aliar sensibilidade a variações de pressão e/ou temperatura com as demais, já citadas, características das fibras.
Entre as mais diversas possibilidades de polímeros capazes de modificar eficientemente a superfície de fibras naturais, como a do coco, merece destaque a polianilina, PAni. A PAni é um polímero condutor típico, resultante da polimerização oxidativa da anilina, cuja condutividade pode ser afetada pelo grau de dopagem, pelo tipo de dopante, pela morfologia e pelo grau de cristalização ${ }^{[5,6]}$.

A PAni tem atraído muita atenção nas últimas décadas devido a suas características como baixo custo, alta condutividade e boa resistência ao ambiente, além de propriedades eletrônicas especiais que podem ser controladas reversivelmente pelos processos de protonação / desprotonação do material ${ }^{[7-10]}$. Além disso, a PAni possui grande potencial para aplicações de ponta, tais como eletrodos em baterias ${ }^{[11]}$, na microeletrônica ${ }^{[12]}$, como materiais eletrocrômicos usados em visores ${ }^{[13]}$, em sensores ${ }^{[14-16]}$, como blindagem eletromagnética ${ }^{[17]}$, entre outras.

Assim, a modificação das fibras de coco é vantajosa sob uma série de aspectos, entre os quais estão o incentivo a um maior aproveitamento destas fibras naturais, além da transferência das atrativas propriedades elétricas ${ }^{[18-24]}$ da polianilina para a fibra, que poderia ser utilizada em diversos tipos de sensores, como os de pressão, extensão e até quimiométricos. Isto tudo, com o uso de quantidades mínimas de polianilina na forma de um nano-recobrimento condutor. Este tipo de recobrimento é factível, conforme demonstrado em publicações recentes do grupo ${ }^{[23-26]}$, onde conseguimos produzir estruturas nanométricas, com diâmetro médio inferior a $50 \mathrm{~nm}$, determinado por espalhamento de raios $\mathrm{X}$ em baixos ângulos (SAXS). Estas nano-estruturas desempenham 
um papel singular na modificação das propriedades elétricas e eletromecânicas das misturas condutoras, em comparação com polianilinas convencionalmente obtidas e de dimensões superiores às nanométricas, demonstrando a importância do controle do tamanho das partículas condutoras, inserindo a presente proposta na área de nanotecnologia.

No campo de sensores de pressão, a fibra de coco recoberta com polianilina pode ter diversas utilidades, entre as quais a de maior destaque seria a utilização deste tipo de material como substituto de parte do estofamento de fibras de coco não modificadas, transformando assentos e colchões em dispositivos inteligentes, capazes de ajustar, em conjunto com outros equipamentos, por exemplo, a temperatura de um veículo ou de um dormitório. Este simples exemplo teria grande impacto sobre as indústrias automobilística e médica, no que tange ao aumento do conforto dos respectivos usuários. Assim, novas tecnologias poderiam ser desenvolvidas ou barateadas e universalmente difundidas, contribuindo para a melhoria da qualidade de vida e preservação ambiental.

\section{Experimental}

\section{Materiais}

A fibra de coco foi gentilmente doada pelo "Projeto Coco Verde Reciclado". Ácido sulfúrico, anilina (Ani), ambos procedentes da VETEC Química Fina, usados como recebido. Persulfato de amônio (APS), Quimibrás Indústrias Químicas S/A também usado como recebido.

\section{Modificação da fibra}

A fibra foi desembaraçada e picotada em tamanho de $10 \mathrm{~cm}$ de comprimento. Após pesadas, as amostras foram submetidas à secagem sob vácuo dinâmico durante 24 horas. A fibra de coco é um material hidrofílico ${ }^{[1-3,27,28]}$. Polímeros polares como a polianilina ${ }^{[29]}$ podem ser efetivamente conectados a superfície de fibras vegetais hidrofílicas, conforme demonstrado em trabalhos anteriores do grupo $^{[30,31]}$. Buscando isso, porções conhecidas das fibras de coco (aproximadamente $10 \mathrm{~g}$ ) foram tratadas em uma solução comercial de hipoclorito de sódio, visando diminuir a quantidade de lignina presente nas fibras. Esse tratamento é importante dada a competição pelo oxidante entre a lignina e o monômero ${ }^{[30,31]}$. Após esta etapa, as fibras foram lavadas e transferidas para um reator de $1 \mathrm{~L}$. A este reator foram adicionados $600 \mathrm{~mL}$ de uma solução de $\mathrm{H}_{2} \mathrm{SO}_{4} 1 \mathrm{M}$. Em seguida, foram acrescentados diferentes quantidades de anilina e de persulfato de amônio, sempre em razão equimolar. As quantidades de anilina adicionadas são mostradas na Tabela 1 . Todas as reações foram feitas em triplicata.

\section{Caracterização das fibras de coco modificadas}

A fibra de coco e a fibra de coco modificada foram avaliadas por microscopia FTIR usando o microscópio FTIR iN10 da Nicolet. Nas análises de infravermelho, as amostras foram acondicionadas dentro de um dessecador e em seguida levadas para o ensaio. $\mathrm{O}$ equipamento foi configurado para realizar 100 varreduras com resolução de $4 \mathrm{~cm}^{-1}$. Esta caracterização teve por objetivo avaliar a fibra não modificada e determinar a constituição química da superfície da fibra por meio de espectros e imagens. As dispersões de PAni resultantes dos processo de modificação das fibras de coco foram investigadas por UV-Vis, buscando informações relevantes sobre a extensão do grau de dopagem da polianilina nestes materiais. Os materiais preparados também foram estudados por WAXS e por SAXS buscando determinar o tamanho dos nano-agregados.

\section{Ensaios elétricos}

A resistividade volumétrica das fibras foi avaliada usando a técnica dos três eletrodos, entre os quais uma quantidade conhecida de fibra modificada era imobilizada com o auxílio de uma luva de teflon. Os testes foram feitos utilizando um multímetro de bancada ICEL MD - 6700 .

As sensibilidades à compressão de uma massa conhecida de fibras de coco modificadas foram avaliadas seguindo metodologia proposta anteriormente pelo grupo ${ }^{[29]}$, usando o arranjo experimental apresentado na Figura 1.

De uma maneira um pouco mais abrangente, para a realização destes ensaios, foi construído um porta-amostras para medidas de resistividade elétrica capaz de suportar as condições de compressão aplicadas ao longo do procedimento. Este porta-amostras foi conectado a um multímetro Multímetro ICEL 6700. Após a conexão, o porta-amostras é acondicionando entre os pratos da máquina de ensaios universais EMIC DL 2000. A máquina de ensaios foi

Tabela 1. Quantidades de anilina e de persulfato de amônio.

\begin{tabular}{ccc}
\hline Condição & Ani (mol) & APS $(\mathbf{m o l})$ \\
\hline 1 & $5 \times 10^{-3}$ & $5 \times 10^{-3}$ \\
2 & $1,1 \times 10^{-2}$ & $1,1 \times 10^{-2}$ \\
3 & $1,6 \times 10^{-2}$ & $1,6 \times 10^{-2}$ \\
4 & $2,2 \times 10^{-2}$ & $2,2 \times 10^{-2}$ \\
5 & $2,7 \times 10^{-2}$ & $2,7 \times 10^{-2}$ \\
6 & $3,3 \times 10^{-2}$ & $3,3 \times 10^{-2}$ \\
\hline
\end{tabular}

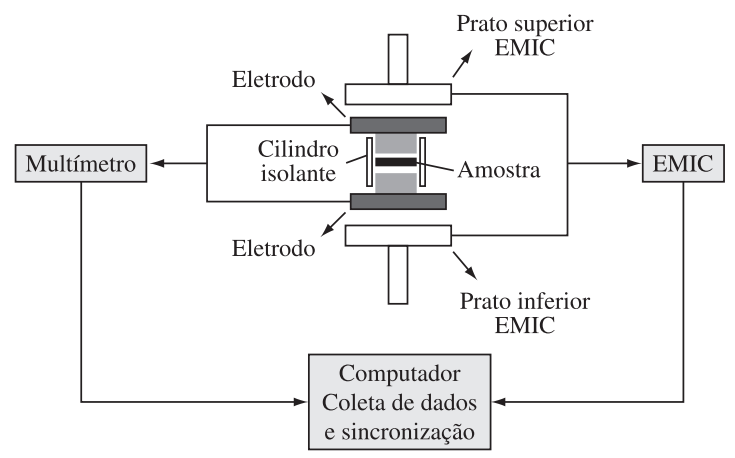

(a)
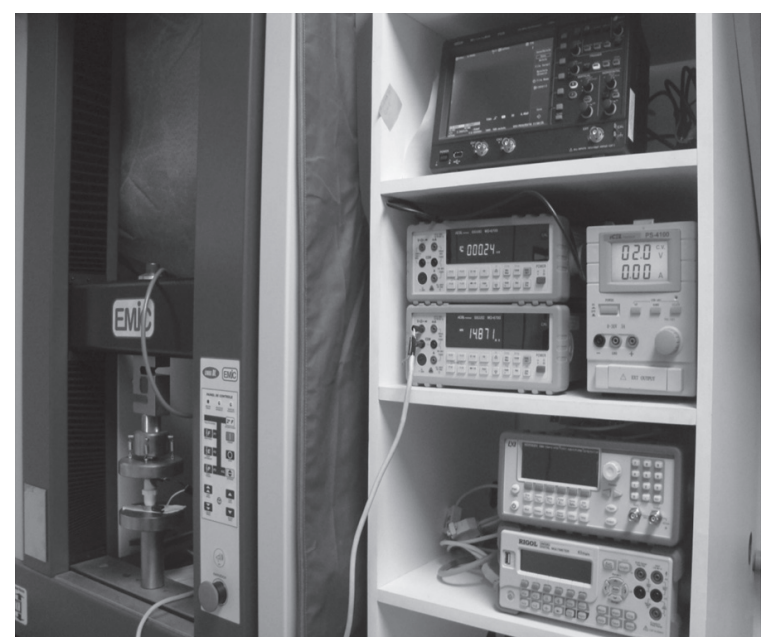

(b)

Figura 1. Esquema (a) e foto (b) do sistema usado para medidas de sensibilidade à compressão. 
ajustada para exercer uma força máxima de $2 \mathrm{kN}$. A velocidade de ensaio foi ajustada em $2 \mathrm{~mm} / \mathrm{s}$ e o número de ciclos de compressão seguida de descompressão foi igual a 10. Todos os ensaios foram realizados em triplicata.

Os dados provenientes da máquina de ensaios e do multímetro foram registrados, sincronizados e tratados por meio do de um programa computacional desenvolvido pelo grupo ${ }^{[29,30]}$. Primeiramente, o programa eletromecânico compara os tempos dos dois equipamentos, registrados em seus respectivos arquivos de relatórios de dados. O critério utilizado para a sincronização é a diferença entre os tempos registrados pelos equipamentos. Ou seja, se a diferença entre o tempo registrado pela máquina de ensaios e o tempo registrado pelo multímetro for menor que 0,01 segundo, os dados são considerados simultâneos e registrados em outro arquivo. Caso contrário, são descartados. Em seguida, o programa eletromecânico descarta os pontos onde a força, F, aplicada é menor que $10 \mathrm{~N}$. Neste ponto, $\mathrm{F}=10 \mathrm{~N}$, a resistividade inicial do sistema, $\mathrm{R}_{0}$ é tomada. É calculada então a variação percentual da condutividade, $\Delta \sigma$. Em seguida, os ciclos são separados em suas respectivas curvas de compressão e expansão.

$$
\Delta \sigma=\frac{100 \times\left|R^{-1}-R_{0}^{-1}\right|}{R_{0}^{-1}}
$$

Para a quantificação dos resultados, o programa computacional calcula os valores da sensibilidade a compressão máxima, bem como da pressão onde este máximo ocorre em cada curva analisada. Para o cálculo da histerese foi estipulado que a diferença média entre as variações de condutividade das curvas de carga e de descarga no ponto de aplicação da pressão intermediária entre o valor mínimo e o máximo de força exercida pela máquina de ensaios corresponde à histerese média de cada sistema. Assim a histerese média, $\overline{\mathrm{h}}$, foi definida de acordo com a Equação 2.

$$
\bar{h}=\sum_{j=1}^{n} \frac{(d S c(P m)-d S d(P m))}{n}
$$

Na Equação 2, “ $n$ ” corresponde ao número de ciclos de carga e descarga a que o material foi submetido; "dSc" e "dSd" são as variações de condutividade referentes aos semi-ciclos de carga e de descarga, respectivamente e Pm é a pressão intermediária aplicada. Ao término destes cálculos um relatório é gerado. Neste relatório são reportados os nomes das amostras testadas e todas as respectivas propriedades eletromecânicas mencionadas.

\section{Resultados}

\section{Modificação da superfície da fibra de coco}

A modificação das fibras de coco com polianilina seguiu o planejamento proposto na Tabela 1. As fibras foram pesadas antes e após o processo e por diferença de massa foi determinado a fração de PAni sobre as fibras. Esses valores são mostrados na Tabela 2.
Os resultados da Tabela 2 são mostrados na forma de um gráfico na Figura 2.

A Figura 2 mostra que, no intervalo estudado, a quantidade de PAni sobre a fibra está linearmente relacionada $\left(\mathrm{R}^{2}=0,9694\right)$ com quantidade de anilina (e consequentemente de APS) empregada na síntese. Assim é possível ajustar com relativa facilidade a quantidade de recobrimento condutor sobre a fibra, o que em teoria permite o ajuste fino de algumas propriedades como a condutividade dos materiais.

\section{Caracterização da fibra de coco modificada}

A Figura 3 mostra as micrografias ópticas da fibra de coco e da fibra modificada segundo a condição 1 apresentada na Tabela 1 .

O material não modificado apresenta coloração amarela característica da fibra de coco enquanto que a fibra modificada com PAni. $\mathrm{H}_{2} \mathrm{SO}_{4}$ apresenta uma coloração esverdeada, característica da PAni. A microscopia óptica da fibra coco modificada também permite inferir que o recobrimento da PAni sobre a fibra é de natureza contínua porém não uniforme. Esse resultado morfológico é um indicativo da baixa quantidade de anilina necessária ao processo de recobrimento da fibra vegetal, o que garante um caráter ecologicamente amigável ao produto final. Além disso, é importante destacar que o resultado de microscopia óptica é um forte indício de que o processo de modificação foi bem sucedido e de que o material, devido à formação de pontos condutores contínuos ao longo da fibra, apresentará boa condutividade.

As análises de microscopia-FTIR foram usadas para o estudo da modificação da superfície da fibra de coco pela PAni. $\mathrm{H}_{2} \mathrm{SO}_{4}$. Os espectros da fibra de coco pura e da fibra modificada são apresentados nas Figuras 4 e 5.

A caracterização da fibra de coco por FTIR, Figura 4, mostra uma banda larga na região de $3500 \mathrm{~cm}^{-1}$ relativa à deformação axial do grupo $\mathrm{OH}$. Esta banda tem este formato largo devido as ligações de hidrogênio. O dublete ao redor de $2900 \mathrm{~cm}^{-1}$ corresponde à deformação axial da ligação $\mathrm{C}-\mathrm{H}$. A banda característica próxima de

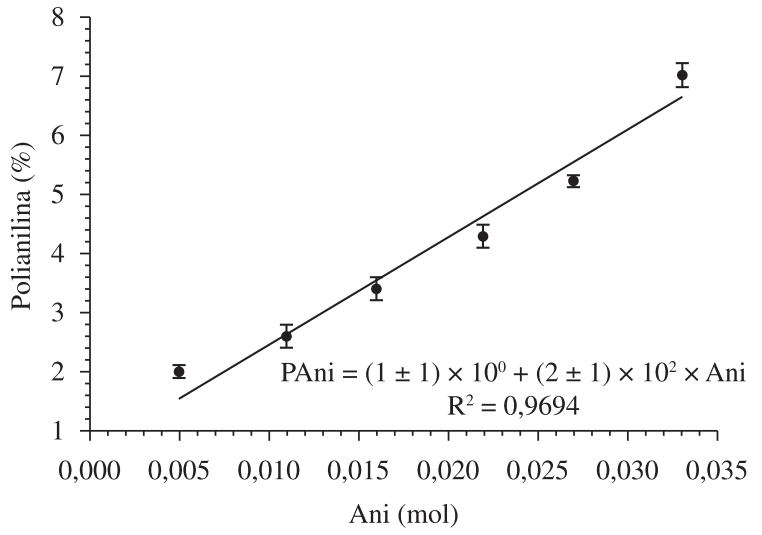

Figura 2. PAni sobre as fibras vegetais em função da quantidade de anilina.

Tabela 2. Percentual de PAni sobre as fibras de coco.

\begin{tabular}{cccccc}
\hline Condição & $\begin{array}{c}\text { Ani } \\
(\mathbf{m o l})\end{array}$ & $\begin{array}{c}\text { APS } \\
(\mathbf{m o l})\end{array}$ & mi $(\mathbf{g})$ & mf (g) & $\begin{array}{c}\text { PAni } \\
(\%)\end{array}$ \\
\hline 1 & $5 \times 10^{-3}$ & $5 \times 10^{-3}$ & $10,0251 \pm 0,0521$ & $10,2297 \pm 0,0462$ & $2,0 \pm 0,1$ \\
2 & $1,1 \times 10^{-2}$ & $1,1 \times 10^{-2}$ & $10,6451 \pm 0,0481$ & $10,9248 \pm 0,0268$ & $2,6 \pm 0,2$ \\
3 & $1,6 \times 10^{-2}$ & $1,6 \times 10^{-2}$ & $10,4598 \pm 0,0368$ & $10,8280 \pm 0,0580$ & $3,4 \pm 0,2$ \\
4 & $2,2 \times 10^{-2}$ & $2,2 \times 10^{-2}$ & $10,2254 \pm 0,0667$ & $10,6848 \pm 0,0497$ & $4,3 \pm 0,2$ \\
5 & $2,7 \times 10^{-2}$ & $2,7 \times 10^{-2}$ & $10,5541 \pm 0,0794$ & $11,1330 \pm 0,0752$ & $5,2 \pm 0,1$ \\
6 & $3,3 \times 10^{-2}$ & $3,3 \times 10^{-2}$ & $10,2144 \pm 0,0257$ & $10,9832 \pm 0,0467$ & $7,0 \pm 0,2$ \\
\hline
\end{tabular}


$1700 \mathrm{~cm}^{-1}$ está relacionada com a deformação axial do grupo $\mathrm{C}=\mathrm{O}$ ácido enquanto que a banda característica em cerca de $1550 \mathrm{~cm}^{-1}$ está relacionada com a ligação $\mathrm{C}=\mathrm{O}$ do aldeído conjugada com a ligação $\mathrm{C}=\mathrm{C}$. A banda característica próxima de 1200 corresponde a deformação axial assimétrica C-O-C $\mathrm{C}^{[31]}$.

A comparação dos espectros de obtidos por microscopiaFTIR das fibras sem recobrimento, Figura 4, e recobertas de PAni, Figura 5, não foi possível devido à coloração esverdeada escura do recobrimento de polianilina, que impediu o processo de reflexão da radiação infravermelha. Por outro lado, a imagem infravermelho da fibra modificada mostra que, na região analisada, há grande

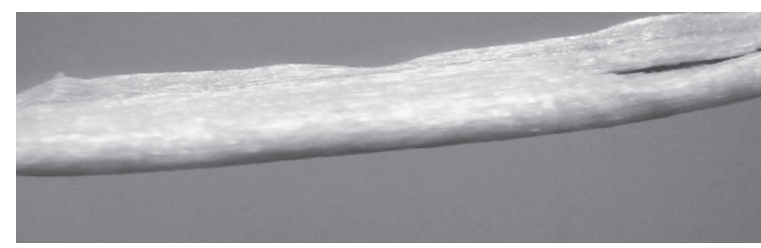

(a)

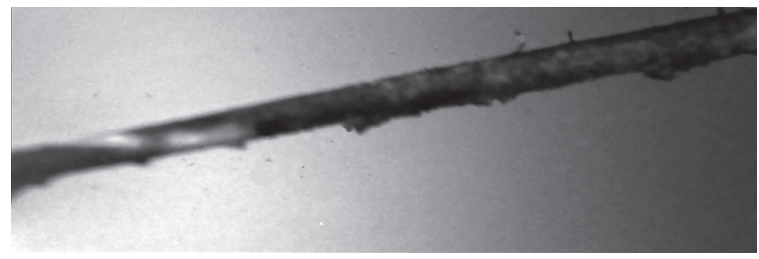

(b)

Figura 3. Fibra de coco (a) e fibra de coco modificada com PAni (b) aumento de $45 \times$.
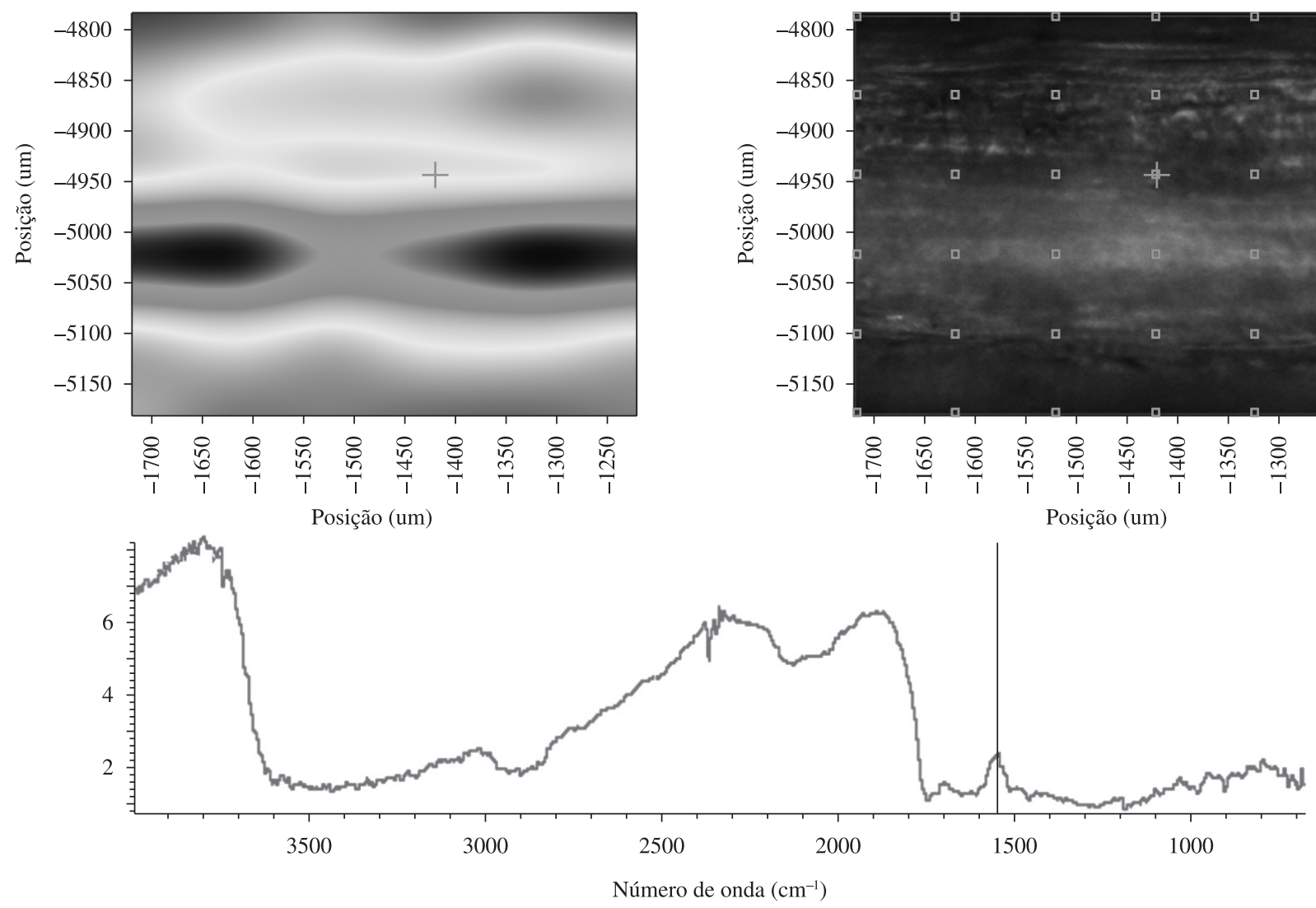

Figura 4. Microscopia FTIR da fibra de coco. absorção de radiação infravermelha sob toda a superfície, indicando que a superfície foi recoberta por uma camada de polianilina.

A espectroscopia de UV-Vis é um importante instrumento na caracterização da polianilina. Em seus espectros são comuns o aparecimento de três bandas. A primeira delas aparece ao redor de $350 \mathrm{~nm}$ (normalmente atribuído à transição das ligações $\varpi \rightarrow \varpi^{*}$ dos anéis benzenóides). Já as outras bandas surgem nas faixas de $400-420$ e $750-800 \mathrm{~nm}$ (atribuídas ao polaron- $\varpi^{*}$ e ao polaron- $\varpi$, respectivamente $)^{[31,32]}$. Na Figura 6 são apresentados os espectros UV-Vis das soluções residuais ácidas contendo Pani. $\mathrm{H}_{2} \mathrm{SO}_{4}$, provenientes dos processos de modificação das fibras de coco nas condições 2,4 e 6 . Nestes espectros são visíveis duas regiões, características dos polarons, ao redor de 400 e $800 \mathrm{~nm}$.

A razão da intensidade das bandas localizadas ao redor de 825 e $320 \mathrm{~nm}$, é um indicativo do grau de dopagem da $\mathrm{PAni}_{2} \mathrm{H}_{2} \mathrm{SO}_{4}$ (Equação 3) ${ }^{[31,32]}$.

$$
\mathrm{Edp}=\mathrm{B} 2 / \mathrm{B} 1
$$

Na Equação 3, B2 é o valor máximo de absorção próximo ao comprimento de onda em $800 \mathrm{~nm}, \mathrm{~B} 1$ é o valor máximo de absorção próximo ao comprimento de onda em $350 \mathrm{~nm}$ e Edp é a estimativa de extensão da dopagem.

Os valores da estimativa de extensão da dopagem (Edp) para as dispersões das fibras preparadas nas condições 2,4 e 6 são iguais a 1,22 $\pm 0,28 ; 1,28 \pm 0,26$; e 1,44 $\pm 0,33$, respectivamente. Estes resultados superiores a 1 indicam a presença de polarons provenientes da protonação da polianilina ${ }^{[31,32]}$. Os valores das razões obtidas nas Edps estão muito próximos para todas as amostras apesar dos diferentes níveis de absorção para as mesmas faixas de comprimento de onda. Embora a razão entre a concentração de anilina e de APS tenha sido mantida contante, entre as amostras

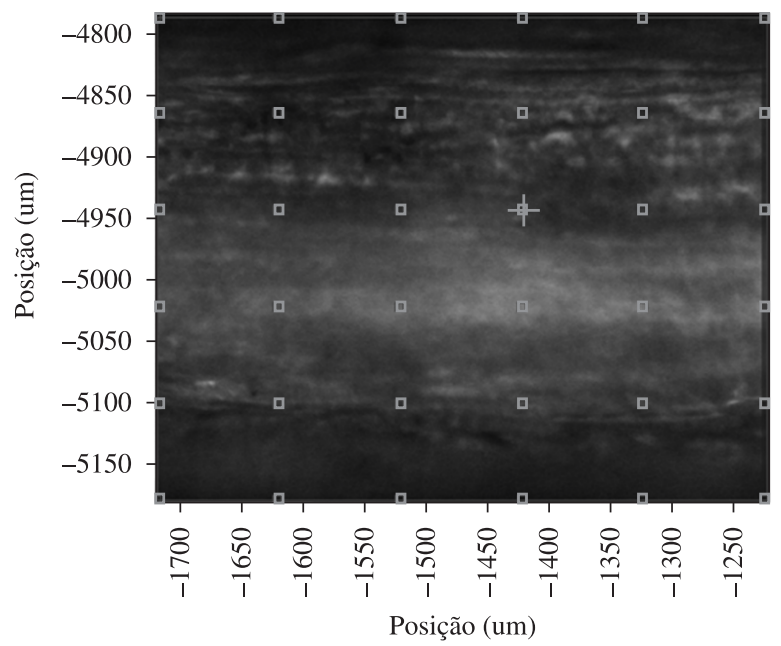



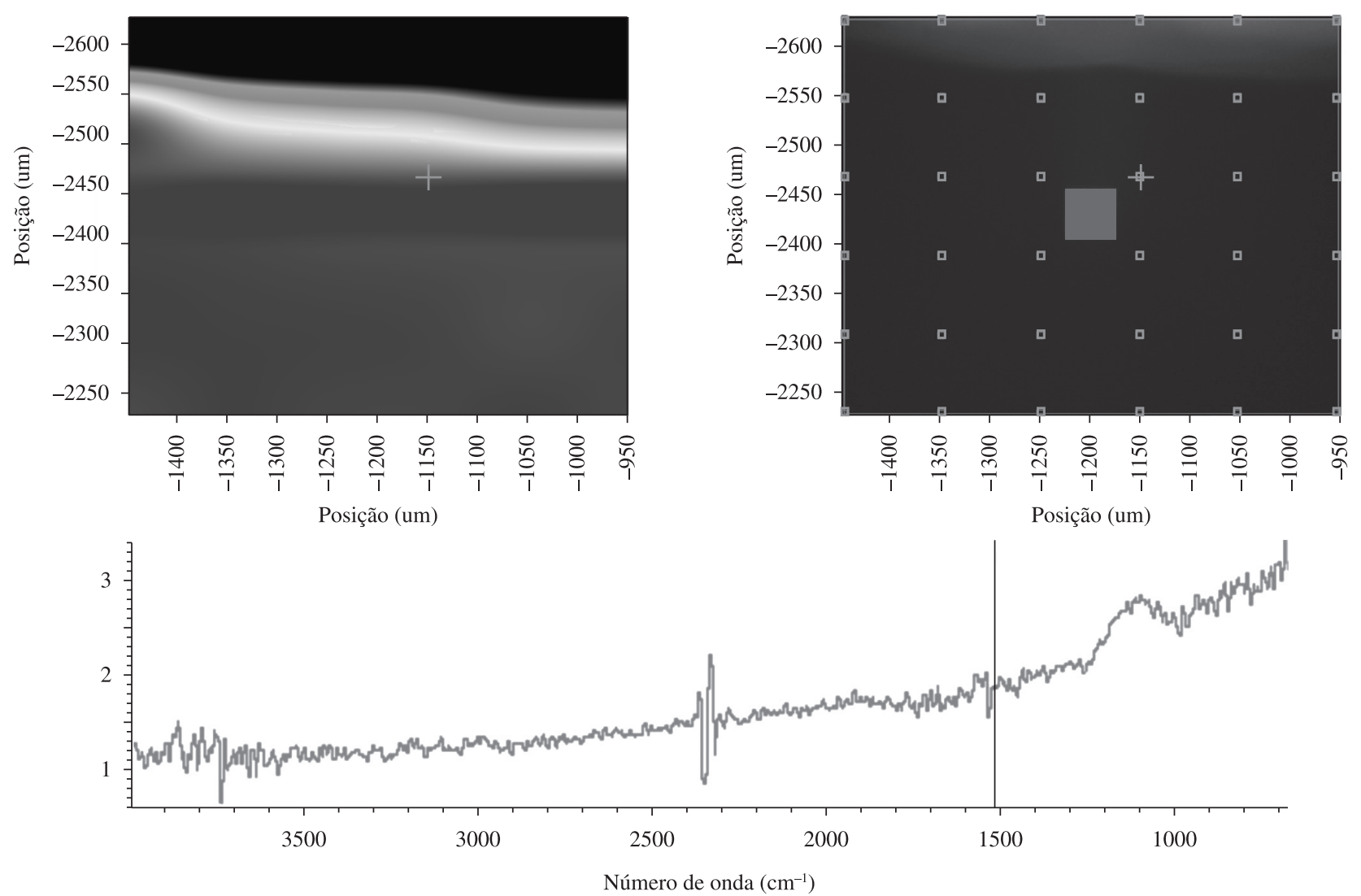

Figura 5. Microscopia FTIR da fibra de coco modificada com PAni.

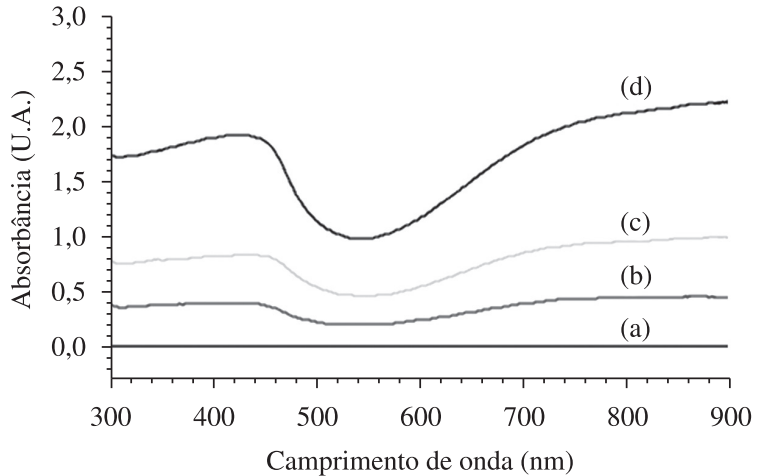

Figura 6. UV-Vis do branco (a) e das amostras obtidas nas condições $2 b, 4 c$ e $6 \mathrm{~d}$, detalhadas na Tabela 1.

estudadas, a amostra preparada na condição 6 apresentou o maior valor de extensão de dopagem. Este resultado sugere que essa condição gerou o material com a maior concentração de polarons e, possivelmente, de maior condutividade.

Os difratogramas da fibra pura e das fibras modificadas com diferentes quantidades de anilina são mostrados na Figura 7.

A PAni. $\mathrm{H}_{2} \mathrm{SO}_{4}$ apresenta célula unitária pseudo-ortorrômbica caracterizada por picos de difração em valores de $2 \theta$ iguais a 20,2; 21,$6 ; 26,7 ; 29,8$ e $34,6^{\circ}$, Contudo, possivelmente devido a elevada intensidade da difração das fibras puras associada com a reduzida fração de polímero condutor sobre as fibras, não foi possível observar os citados picos nos materiais modificados com PAni.

A cristalinidade das fibras foi calculada seguindo o método de Ruland ${ }^{[33]}$. Os resultados obtidos são mostrados na Tabela 3.

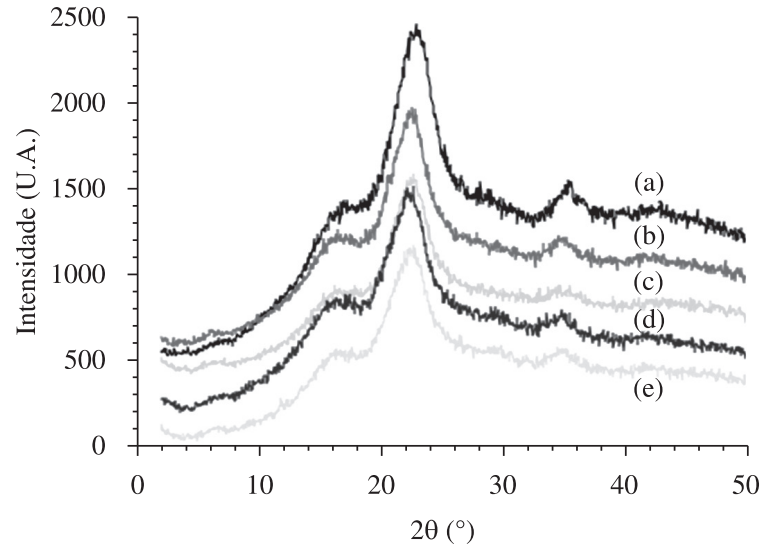

Figura 7. Difratogramas da fibra pura (a) e das fibras modificadas de acordo com as condições 1b, 2c, 4d e 6e, detalhadas na Tabela 1.

É importante destacar que os resultados de difração de raios $\mathrm{X}$ mostram que o processo de modificação das fibras não provoca perdas consideráveis da cristalinidade do material, indicando que a fração celulósica não é afetada pelo processo de síntese.

O perfil SAXS obtido por subtração do espalhamento da fibra pura do espalhamento da fibra de coco modificada com cerca de $7 \%$ de PAni. $\mathrm{H}_{2} \mathrm{SO}_{4}$, mostrado na Figura 8, revela a presença de picos de correlação espacial na região entre 0,4 e $0,8 \mathrm{~nm}^{-1}$. Este sinal é resolvido por meio da deconvolução do espalhamento em três picos gaussianos, que geram um envelope com correlação 0,962 , em relação aos pontos experimentais.

A presença dos picos apresentados na Figura 8 indica a existência de heterogeneidades espaciais de tamanho nanométrico ${ }^{[23,34]}$. Os 
Tabela 3. Cristalinidade da fibra de coco e das fibras modificadas com Pani.

\begin{tabular}{cc}
\hline PAni $(\boldsymbol{\%})$ & Cristalinidade (\%) \\
\hline 0 & $53 \pm 3$ \\
$2,0 \pm 0,1$ & $52 \pm 2$ \\
$2,6 \pm 0,2$ & $54 \pm 4$ \\
$4,3 \pm 0,2$ & $56 \pm 2$ \\
$7,0 \pm 0,2$ & $49 \pm 4$ \\
\hline
\end{tabular}

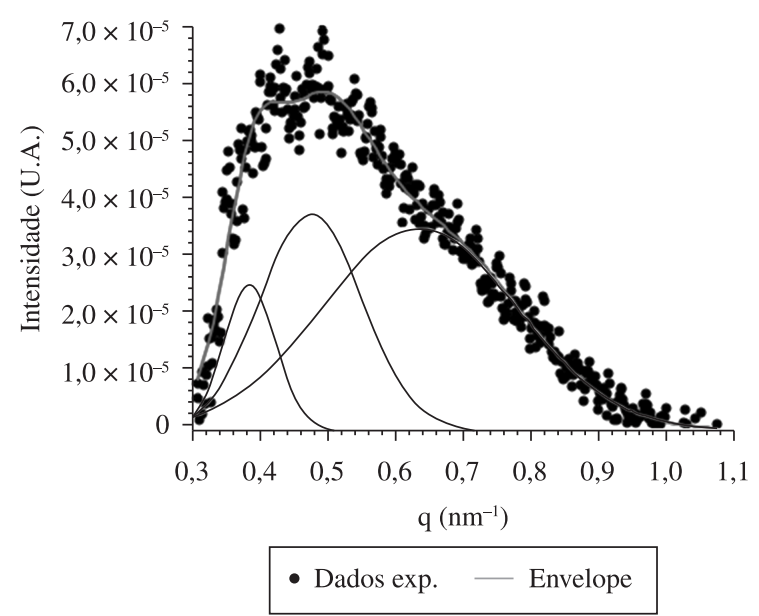

Figura 8. Deconvolução gaussiana do espalhamento da fibra de coco modificada com cerca de $7 \%$ de PAni.

dados obtidos a partir da deconvolução gaussiana destes picos são mostrados na Tabela 4.

A distância média mais provável $\left(\mathrm{d}_{\mathrm{S}}\right)$ entre as heterogeneidades pode ser estimada de acordo com a Equação $3^{[35]}$ :

$$
d_{S}=2 \pi / q_{\max }
$$

Na Equação 3, $q_{\max }$ é o módulo do vetor espalhamento no máximo do pico. As heterogeneidades que produzem o espalhamento de raios $\mathrm{X}$ na $\mathrm{PAni} . \mathrm{H}_{2} \mathrm{SO}_{4}$ estão relacionadas com as diferenças de densidade eletrônica causadas pela existência de regiões cristalinas nanometricamente ordenadas, dispersas sobre a fibra ${ }^{[23]}$. Os valores de $\mathrm{d}_{\mathrm{S}}$ calculados são também mostrados na Tabela 4 .

$\mathrm{O}$ tamanho médio destas heterogeneidades, $\mathrm{L}_{\mathrm{C}}$, provê uma estimativa do tamanho do "super-cristal" desordenado e é calculado de acordo com a Equação $4^{[35]}$ :

$$
L_{C}=4 \pi / \Delta q
$$

$\mathrm{Na}$ Equação 4, $\Delta q$ é a largura a meia altura (FWHM) do pico. Os valores de $\mathrm{L}_{\mathrm{C}}$ calculados são também mostrados na Tabela 4.

A PAni. $\mathrm{H}_{2} \mathrm{SO}_{4}$ que recobre as fibras de coco apresentou três picos. O primeiro pico, centrado ao redor de $3,86 \times 10^{-1} \mathrm{~nm}^{-1}$, apresenta valor de $\mathrm{L}_{\mathrm{c}}$ igual a $(163 \pm 19) \mathrm{nm}$ e valor de $\mathrm{dS}$ igual a $(16,3 \pm 0,2) \mathrm{nm}$. Por sua vez, o segundo pico, centrado ao redor de $4,76 \times 10^{-1} \mathrm{~nm}^{-1}$, apresenta valor de $\mathrm{L}_{\mathrm{c}}$ igual a $(83 \pm 10) \mathrm{nm}$ e valor de dS igual a $(13,2 \pm 0,1) \mathrm{nm}$. Já o terceiro pico, centrado ao redor de $6,36 \times 10^{-1} \mathrm{~nm}^{-1}$, apresenta valor de $\mathrm{L}_{\mathrm{c}}$ igual a $(44 \pm 4) \mathrm{nm}$ e valor de $\mathrm{d}_{\mathrm{s}}$ igual a $(9,9 \pm 0,1) \mathrm{nm}$. A existência de três conjuntos distintos de valores de $\mathrm{L}_{\mathrm{c}}$ e de $\mathrm{d}_{\mathrm{s}}$ indica a presença de diferentes cristais de PAni. $\mathrm{H}_{2} \mathrm{SO}_{4}$, o que está de acordo com outros resultados reportados na literatura científica ${ }^{[23,35-38]}$. Assim, os resultados de SAXS são um forte indício da obtenção de nano-partículas de PAni sobre a superfície das fibras de coco.
Tabela 4. Dados obtidos via deconvolução do perfil 1D do SAXS da fibra de coco modificada com cerca de $7 \%$ de PAni.

\begin{tabular}{ccccc}
\hline Pico & Centro $\left(\mathbf{n m}^{-1}\right)$ & Largura $\left(\mathbf{n m}^{-1}\right)$ & $\mathbf{d}_{\mathbf{s}}(\mathbf{n m})$ & $\mathbf{L}_{\mathbf{c}}(\mathbf{n m})$ \\
\hline 1 & $(3,86 \pm 0,04) \times 10^{-1}$ & $(7,7 \pm 0,2) \times 10^{-2}$ & $16,3 \pm 0,2$ & $163 \pm 19$ \\
2 & $(4,76 \pm 0,06) \times 10^{-1}$ & $(1,5 \pm 0,2) \times 10^{-2}$ & $13,2 \pm 0,1$ & $83 \pm 10$ \\
3 & $(6,36 \pm 0,05) \times 10^{-1}$ & $(2,9 \pm 0,3) \times 10^{-2}$ & $9,9 \pm 0,1$ & $44 \pm 4$ \\
\hline
\end{tabular}

Os resultados calculados de resistividade volumétrica são apresentados na Tabela 5. A fibra de coco não modificada apresentou valores de resistividade da ordem de $10^{7} \mathrm{Ohm} . \mathrm{cm}$ enquanto as fibras modificadas com as menores quantidades de PAni, ao redor de $2 \%$, apresentaram valores de resistividade da ordem de $10^{3} \mathrm{Ohm}$. $\mathrm{cm}$. Quantidades maiores de polianilina produziram diminuições ainda maiores da resistividade dos materiais de modo que as fibras modificadas com 2,0; 2,6; 3,4; 4,3; 5,2 e 7,0\% de PAni apresentaram aumentos de condutividade de cerca de 46.000; 60.000; 320.000; 290.000; $920.000 ; 1.800 .000$ vezes, respectivamente.

O aumento de condutividade das fibras modificadas já com pequenas quantidades de PAni permite a fácil utilização destas fibras como componentes resistivos em circuitos elétricos sensíveis a variação de temperatura e de pressão, conforme reportado em trabalhos anteriores do grupo ${ }^{[16,29,31,39]}$.

A resistividade das fibras modificadas com PAni em função da pressão foi estudada e os resultados das triplicatas também são mostrados na Tabela 5. Já a Figura 9 mostra a variação de condutividade em função da pressão aplicada para algumas das amostras testadas. Os resultados da Tabela 5 permitiram calcular os dados médios de variação da condutividade em função da pressão, apresentados na Tabela 6 .

Entre as amostras estudadas, a fibra de coco não modificada apresentou, conforme esperado, os menores valores de variação de condutividade ao longo dos semi-ciclos de compressão e de descompressão. A variação de condutividade observada para as fibras de coco não modificadas está relacionada com uma maior compactação das fibras, que gera um melhor contato entre as fibras e o eletrodo, diminuindo assim a resistividade medida.

Por outro lado, as amostras modificadas com polianilina apresentaram variações da condutividade sobre esforços compressivos muito maiores que as apresentadas pela fibra pura, indicando que a polianilina, além de aumentar a condutividade elétrica, possibilita o uso destas fibras para as aplicações propostas. Assim, as fibras modificadas com 2,0; 2,6; 3,4; 5,2 e 7,0\% de PAni apresentaram variações de condutividade durante o semi-ciclo de compressão ao redor de 4600; 2900; 1700; 1600; 2400 e 3600\%, respectivamente, maiores que a da fibra pura. Esses valores são muito semelhantes aos observados para os mesmos materiais ao longo dos semi-ciclos de expansão.

Desta forma, de acordo com os dados da Tabela 6, as amostras produzidas segundo a condição 1 (recobertas com cerca de $2 \%$ de PAni) são os materiais que apresentam os maiores valores de variação de condutividade $\left((2.0 \pm 1.6) \times 10^{3} \%\right)$, associados com valores de resistividade relativamente baixos (da ordem de $10^{3} \mathrm{Ohm} . \mathrm{cm}$ ), que os tornam, como demonstrado em outros trabalhos do grupo ${ }^{[29,30,39]}$, perfeitamente aplicáveis no preparo de dispositivos sensíveis a variações de pressão. Como vantagem extra, esse material é o que possui a menor quantidade de PAni o que, além de reduzir custos de produção, confere um caráter mais ecologicamente amigável ao mesmo. 


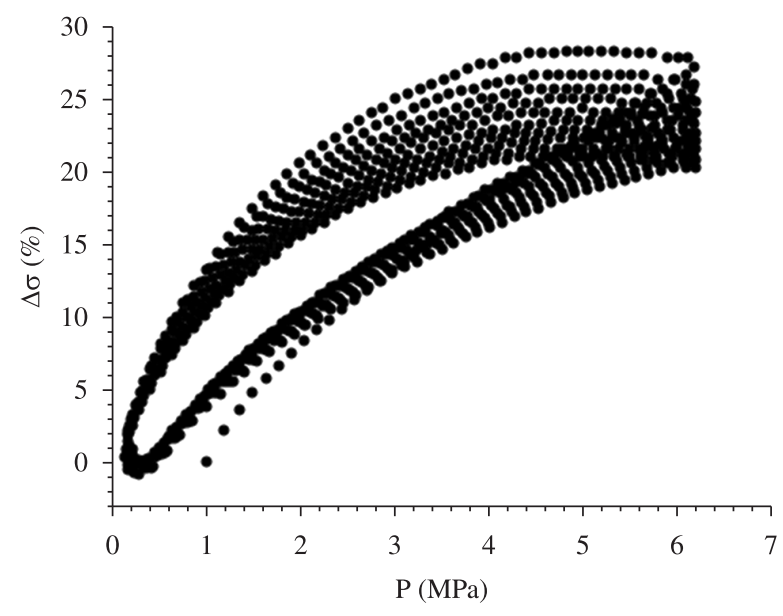

(a)

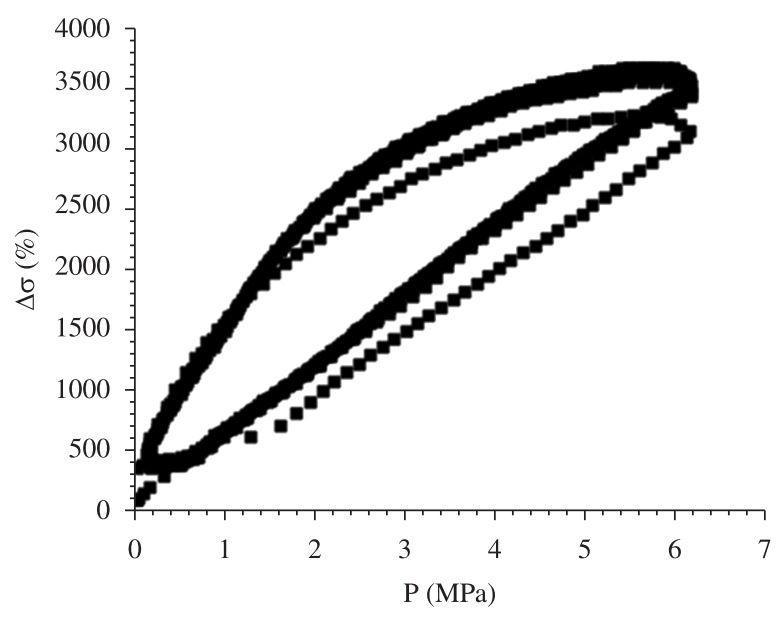

(b)

Figura 9. Variação de condutividade em função da pressão aplicada sobre a fibra de coco (a) e a fibra modificada com cerca de $2,0 \%$ de PAni.

Tabela 5. Conjunto de resultados de resistividade volumétrica e de variação da condutividade em função da pressão aplicada sobre as fibras de coco modificadas com polianilina.

\begin{tabular}{|c|c|c|c|c|}
\hline \multirow[t]{2}{*}{ PAni (\%) } & \multirow{2}{*}{$\begin{array}{c}\text { Resistividade } \\
\text { volumétrica (Ohm.cm) }\end{array}$} & \multicolumn{3}{|c|}{ Variação da condutividade sob pressão } \\
\hline & & $\operatorname{dScMax}(\%)$ & dSdMax $(\%)$ & Hist \\
\hline \multirow{3}{*}{0} & $6,60 \times 10^{7}$ & $(5,1 \pm 0,5) \times 10^{1}$ & $(5,2 \pm 0,5) \times 10^{1}$ & $(0,3 \pm 0,6) \times 10^{1}$ \\
\hline & $8,30 \times 10^{7}$ & $(5,9 \pm 0,5) \times 10^{1}$ & $(6,0 \pm 0,6) \times 10^{1}$ & $(0,8 \pm 1,5) \times 10^{1}$ \\
\hline & $7,10 \times 10^{7}$ & $(2,4 \pm 0,2) \times 10^{1}$ & $(2,5 \pm 0,3) \times 10^{1}$ & $(0,7 \pm 1,3) \times 10^{1}$ \\
\hline \multirow{3}{*}{$2,0 \pm 0,1$} & $1,50 \times 10^{3}$ & $(2,4 \pm 0,2) \times 10^{3}$ & $(2,4 \pm 0,2) \times 10^{3}$ & $(5 \pm 1) \times 10^{2}$ \\
\hline & $1,60 \times 10^{3}$ & $(3,5 \pm 0,1) \times 10^{3}$ & $(3,6 \pm 0,1) \times 10^{3}$ & $(1,20 \pm 0,03) \times 10^{3}$ \\
\hline & $1,70 \times 10^{3}$ & $(2,8 \pm 0,3) \times 10^{2}$ & $(2,9 \pm 0,2) \times 10^{2}$ & $(5,3 \pm 0,3) \times 10^{1}$ \\
\hline \multirow{3}{*}{$2,6 \pm 0,2$} & $8,30 \times 10^{2}$ & $(9 \pm 2) \times 10^{2}$ & $(10 \pm 2) \times 10^{2}$ & $(2,3 \pm 0,3) \times 10^{2}$ \\
\hline & $9,20 \times 10^{2}$ & $(1,1 \pm 0,4) \times 10^{3}$ & $(1,2 \pm 0,4) \times 10^{3}$ & $(2 \pm 1) \times 10^{2}$ \\
\hline & $1,90 \times 10^{3}$ & $(1,8 \pm 0,2) \times 10^{3}$ & $(1,8 \pm 0,2) \times 10^{3}$ & $(3,3 \pm 0,8) \times 10^{2}$ \\
\hline \multirow{3}{*}{$3,4 \pm 0,2$} & $2,20 \times 10^{2}$ & $(1,1 \pm 0,2) \times 10^{3}$ & $(1,1 \pm 0,3) \times 10^{3}$ & $(2,2 \pm 0,5) \times 10^{2}$ \\
\hline & $2,30 \times 10^{2}$ & $(4 \pm 1) \times 10^{2}$ & $(4 \pm 1) \times 10^{2}$ & $(7 \pm 4) \times 10^{1}$ \\
\hline & $2,30 \times 10^{2}$ & $(8 \pm 4) \times 10^{2}$ & $(9 \pm 4) \times 10^{2}$ & $(2 \pm 1) \times 10^{2}$ \\
\hline \multirow{3}{*}{$4,3 \pm 0,2$} & $1,90 \times 10^{2}$ & $(5 \pm 3) \times 10^{2}$ & $(5 \pm 3) \times 10^{2}$ & $(7 \pm 4) \times 10^{1}$ \\
\hline & $2,10 \times 10^{2}$ & $(1,3 \pm 0,2) \times 10^{3}$ & $(1,3 \pm 0,2) \times 10^{3}$ & $(1,2 \pm 0,5) \times 10^{2}$ \\
\hline & $3,60 \times 10^{2}$ & $(3,0 \pm 0,5) \times 10^{2}$ & $(3,0 \pm 0,5) \times 10^{2}$ & $(3 \pm 2) \times 10^{1}$ \\
\hline \multirow{3}{*}{$5,2 \pm 0,1$} & $4,20 \times 10^{1}$ & $(7 \pm 1) \times 10^{2}$ & $(7 \pm 1) \times 10^{2}$ & $(1,1 \pm 0,3) \times 10^{2}$ \\
\hline & $4,60 \times 10^{1}$ & $(1,0 \pm 0,1) \times 10^{3}$ & $(1,0 \pm 0,1) \times 10^{3}$ & $(1,2 \pm 0,3) \times 10^{2}$ \\
\hline & $1,50 \times 10^{2}$ & $(1,5 \pm 0,2) \times 10^{3}$ & $(1,5 \pm 0,2) \times 10^{3}$ & $(2 \pm 1) \times 10^{2}$ \\
\hline \multirow{3}{*}{$7,0 \pm 0,2$} & $2,80 \times 10^{1}$ & $(8 \pm 1) \times 10^{2}$ & $(8 \pm 1) \times 10^{2}$ & $(1,2 \pm 0,2) \times 10^{2}$ \\
\hline & $4,10 \times 10^{1}$ & $(1,9 \pm 0,3) \times 10^{3}$ & $(2,0 \pm 0,3) \times 10^{3}$ & $(3 \pm 1) \times 10^{2}$ \\
\hline & $5,10 \times 10^{1}$ & $(2,2 \pm 0,4) \times 10^{3}$ & $(2,2 \pm 0,4) \times 10^{3}$ & $(3 \pm 1) \times 10^{2}$ \\
\hline
\end{tabular}

Tabela 6. Variação média da condutividade em função da pressão e da quantidade percentual de PAni sobre as fibras.

\begin{tabular}{cccc}
\hline PAni $(\%)$ & dScMa $\times(\%)^{*}$ & dSdMa $\times(\%)^{*}$ & Histerese $(\%)$ \\
\hline 0 & $(4,5 \pm 1,8) \times 10^{1}$ & $(4,6 \pm 1,8) \times 10^{1}$ & $(6,3 \pm 2,5) \times 10^{1}$ \\
$2,0 \pm 0,1$ & $(2,0 \pm 1,6) \times 10^{3}$ & $(2,1 \pm 1,7) \times 10^{3}$ & $(5,8 \pm 5,8) \times 10^{2}$ \\
$2,6 \pm 0,2$ & $(1,3 \pm 0,5) \times 10^{3}$ & $(1,3 \pm 0,5) \times 10^{3}$ & $(2,7 \pm 0,6) \times 10^{2}$ \\
$3,4 \pm 0,2$ & $(7,6 \pm 3,7) \times 10^{2}$ & $(7,8 \pm 3,7) \times 10^{2}$ & $(1,6 \pm 0,8) \times 10^{2}$ \\
$4,3 \pm 0,2$ & $(6,9 \pm 5,5) \times 10^{2}$ & $(7,0 \pm 5,6) \times 10^{2}$ & $(7,4 \pm 4,3) \times 10^{1}$ \\
$5,2 \pm 0,1$ & $(1,1 \pm 0,4) \times 10^{3}$ & $(1,1 \pm 0,2) \times 10^{3}$ & $(1,4 \pm 0,4) \times 10^{2}$ \\
$7,0 \pm 0,2$ & $(1,6 \pm 0,8) \times 10^{3}$ & $(1,6 \pm 0,8) \times 10^{3}$ & $(2,5 \pm 1,2) \times 10^{2}$ \\
\hline
\end{tabular}

*dScMax e dSdMax são as máximas variações de condutividade durante os semi-ciclos de compressão e de descompressão, respectivamente

\section{Conclusões}

A modificação de fibras de coco com polianilina proposta no presente trabalho é viável. Os resultados obtidos mostraram que é possível obter quantidade controladas do recobrimento condutor sobre a superfície da fibra. Mais do que isso, este recobrimento possui uma coloração esverdeada característica da polianilina, um indicativo da bem sucedida polimerização da anilina sobre as fibras. Estes resultados são apoiados pelos de microscopia-FTIR e pelos de UV-Vis.

Por sua vez, os resultados de SAXS mostram a existência de três conjuntos distintos de valores de $L_{C}$ e de $d_{S}$, indicando a presença de diferentes tipos de estruturas nanométricas de Pani. $\mathrm{H}_{2} \mathrm{SO}_{4}$ sobre a superfície da fibra. 
Outra conclusão muito importante é que as fibras modificadas são capazes de conduzir eletricidade e que essa capacidade de condução está relacionada com a quantidade de PAni sobre as mesmas. Esse resultado é muito encorajador, uma vez que a condutividade elétrica é um pré-requisito para a aplicação de um dado material no campo de sensores resistivos de temperatura e de pressão. Especificamente em relação aos sensores de pressão, as fibras recobertas com cerca de $2 \%$ de PAni são as mais indicadas para aplicações nestes dispositivos, uma vez que apresentam os maiores valores de variação de condutividade, associados com valores de resistividade relativamente baixos. Como vantagem extra, esse material é o que possui a menor quantidade de PAni, o que, além de implicar em menores custos de produção, confere um caráter mais ecologicamente correto ao mesmo.

\section{Agradecimentos}

Os autores agradecem a FAPERJ, a CAPES e ao CNPq pelos recursos para a estruturação do Laboratório de Biopolímeros e Sensores do IMA / UFRJ e pelas bolsas concedidas. Os autores também agradecem ao LNLS pelo suporte nos experimentos de SAXS (LNLS, D11A - SAXS1 \# 7086/08 e \# 9077/10). Um obrigado especial as alunas de iniciação Juliana H. C. Pereira, Liz C. de Aguiar, Cíntia A. da Costa e Amanda de V. Varela pela colaboração.

\section{Referências Bibliográficas}

1. Araujo, A. M.; Rosa, M. F.; Crisóstomo, L. A.; Figueirêdo, M. C. B. \& Cunha, A. E. "Avaliação do potencial de aproveitamento do líquido da casca de coco verde" in: XIX Congresso Brasileiro de Ciência e Tecnologia de Alimentos: Estratégia para o desenvolvimento, RecifePe (2004).

2. Carrijo, O. A.; Liz, R. S. \& Makishima, N. - Hort. Bras., 20, p.533 (2002).

3. Araujo, A. M. - "Tratamento anaeróbio do líquido da casca de coco verde utilizando reator UASB". Dissertação de Mestrado; Universidade Federal do Ceará/Fortaleza (2008).

4. Senhoras, E. M. - "Estratégias de uma agenda para a cadeia agroindustrial do coco: transformando a ameaça dos resíduos em oportunidades eco-eficientes", Monografia, Universidade Estadual de Campinas, Instituto de Economia, Campinas (2003).

5. Mattoso, L. H. C. - Quím. Nova, 19, p.388 (1996).

6. Long, Y.; Chen, Z.; Wang, N.; Zhang, Z. \& Wan, M. Physica B, 325, p.208(2003),

7. Galiani, P. D.; Malmonge, J. A.; Santos, D. P. \& Malmonge, L. F. Polímeros, 17, p.93 (2007).

8. Simões, F. R.; Bulhões, L. O. S. \& Pereira, E. C. - Polímeros, 19, p.54 (2009).

9. Gazotti, W. A. Jr. \& De Paoli, M.-A. Synthetic Metals, 80, p.263 (1996).

10. Narkis, M.; Zilberman, M. \& Siegmann, A. - Polym. Adv. Technol., 8, p.525 (1997).

11. Kaneko, M. \& Nakamura, H. - J. Chem. Soc. Commun., 6, p.346 (1985).

12. Chen, S. A. \& Fang, Y. - Synthetic Metals, 60, p.215 (1993).

13. Jelle, B. P.; Hagen, G.; Sunde, S. \& Odegard, R.- Synthetic Metals, 54, p.315 (1993).
14. Grant, L.; Guernion, N.; Ratcliffe, N. M. \& Teare, C. - "Materials Chemistry Conference", Bangor, Abstracts, p.62 (2001).

15. Lee, J. K.; Yoo, D. S.; Handy, E. S. \& Rubner, M. F. - Appl. Phy. Lett., 69, p.1686 (1996).

16. Souza Jr., F. G. - "Desenvolvimento de sensor de pressão a base de elastômeros termoplásticos e polianilina", Tese de Doutorado, IMAUFRJ (2006).

17. Malinauskas, A.- Polymer, 42, p.3957 (2001).

18. Xavier, M. G.; Leite, F. L.; Leite, E. R.; Venancio, E. C.; MacDiarmid, A. G. \& Mattoso, L. H. C. - "NSTI Nanotechnology Conference" (2006).

19. Park, S. Y.; Cho, M. S. \& Choi, H. J. - Curr. Appl. Phys., 4, p.581 (2004).

20. Gao, H.; Jiang, T.; Han, B.; Wang, Y.; Du, J.; Liu, Z. \& Zhang, J. Polymer, 45, p.3017 (2004).

21. Kan, J. Q.; Zhou, S.; Zhang, Y. \& Patel, M. - Eur. Polym. J., 42, p.2004 (2006).

22. Zhao, W.; Ma, L. \& Lu, K. - J. Polym. Res., 14, p.1 (2007).

23. Souza, F. G.; Soares, B. G. \& Dahmouche, K. - J. Polym. Sci. Part B, Polym. Phys., 45, p.3069 (2007).

24. Soares, B. G.; Souza Jr, F. G.; Manjunath, A.; Somashekarappa, H.; Somashekar, R. \& Siddaramaiah. - Pramana, 69, p.435 (2007).

25. Souza Jr, F. G.; Soares, B. G.; Mantovani, G. L.; Manjunath, A.; Somashekarappa, H.; Somashekar, R. \& Siddaramaiah - Mater. Sci. Eng. A, 476, p.240 (2008).

26. Souza Jr, F. G.; Soares, B. G.; Mantovani, G. L.; Manjunath, A.; Somashekarappa, H.; Somashekar, R. \& Siddaramaiah. - Polymer, 47, p.2163 (2006).

27. Gonçalves, C. A. \& Lelis, R. C. C. - Floresta e Ambient., 8, p.167 (2001).

28. Friese, M. A. \& Banerjee, S. - Appl. Spectrosc., 46, p.246 (1992).

29. Souza Jr, F. G.; Michel, R. C. \& Soares, B. G. - Polym. Test., 24, p. 998 (2005).

30. Souza Jr, F. G.. Michel, R. C. \& Soares, B. G. - "Sensor de pressão à base de compósitos condutores", PI 0603379-2 (2006).

31. Souza Jr., F. G.; Oliveira, G. E.; Soares, B. G.; Nele M.; Rodrigues, C. H. M. \& Pinto, J. C. - Macromol. Mater. Eng., 294, p.484 (2009).

32. Souza Jr, F. G.; Pinto, J. C. \& Soares - Eur. Polym. J., 43, p.2007 (2007).

33. Ruland, W. - Acta Cryst., 14, p.1180 (1961).

34. Glatter, O. \& Kratky, O. - "Small-Angle X-ray Scattering", Academic Press, New-York (1982).

35. Lux, F.; Hinrichsen, G.; Krinichnyi, V. I.; Nazarova, I. B.; Cheremisow, S. D. - Pohl. M. M. Synth. Met., 55, p.347 (1993).

36. Kaiser, A. B.; Subramaniam, C. K.; Gilberd, P. W. \& Wessling, B. Synth. Met., 69, p.197 (1995).

37. Berner, D.; Travers, J. P. \& Rannou, P. - Synth. Met., 101, p.836 (1999).

38. Huang, H. G.; Zheng, Z. X.; Luo, J.; Zhang, H. P.; Wu, L. L. \& Lin, Z. H. - Synth. Met., 123, p.321 (2001).

39. Souza Jr, F. G.; Pinto, J. C.; Rodrigues, M. V.; Anzai, T. K.; Richa, P.; Melo Jr, P. A.; Nele, M.; Oliveira, G. E. \& Soares, B. G. - Polym. Eng. Sci., 48, p.1947 (2008).

Enviado: $21 / 04 / 10$

Reenviado: 20/07/10

Aceito: $23 / 07 / 10$

DOI: $10.1590 / \mathrm{S} 0104-14282011005000016$ 